\title{
Power analysis of static VAr compensators
}

\author{
F. R. Quintela*, J. M. G. Arévalo and R. C. Redondo \\ Escuela Técnica Superior de Ingeniería Industrial, Universidad de Salamanca, 37700 Béjar, Spain \\ Email:felixrq@usal.es; jumagar@usal.es; roberrm@usal.es
}

Web: http://www.usal.es/electricidad

NOTICE: This is the author's version of a work accepted for publication by Elsevier. Changes resulting from the publishing process, including peer review, editing, corrections, structural formatting and other quality control mechanisms, may not be reflected in this document. Changes may have been made to this work since it was submitted for publication. A definitive version was subsequently published in International Journal of Electrical Power and Energy Systems, [30, 6/7], July-September 2008, doi:10.1016/j.ijepes.2007.12.002.

\begin{abstract}
Analysis of three-phase loads usually assume them to be three impedances in a star or triangle connection. This is the reason why obtained results can only be considered valid for passive loads, strictly speaking. Analysis leading to the proposal of some static compensators is usually performed in this way, which induces to believe that this compensators are only valid for passive loads. An analysis procedure, which uses only powers to describe loads, is expounded in this paper. If applied to the analysis of static compensators, it reveals unequivocally their usefulness with active and passive threephase loads. Therefore, this method is more general and, as it will be seen, easier.
\end{abstract}

\section{Keywords}

Power analysis, VAr compensator, load balance.

\section{Introduction}

It is usual to suppose three-phase loads exclusively formed by impedances in a star or triangle connection while studying them. For instance, a lot of textbooks do this while deducing the active, reactive, and apparent power's formulas of balanced three-phase systems[1][2]. This deduction method assures the validity of the results for passive loads, but it does not justify their validity for active loads, strictly speaking.

Every three-phase passive load formed only by impedances connected in any manner is equivalent to three impedances in a star or triangle connection. Nevertheless, if the load contains motors or generators it is necessary to describe it by means of voltage or current sources along with impedances. That is to say, the result is an active load, which is not equivalent to a load formed just by three impedances.

Some solutions, which are obtained only for passive loads, are also valid for active loads even though deduction procedures do not justify them. Their apparent limitation is due exclusively to the deduction method. For this reason it is important to use analysis methods that are capable of clearly showing the scope of the solutions they lead to. One of those procedures consists in using relations between the powers of three-phase

\footnotetext{
* Corresponding author. Tel.: +34 923408080 ext.: 2227 Fax: +34 923408127 Email:felixrq@usal.es
} 
systems instead of describing the loads by means of impedances. Three advantages of this method are: a) all variables involved are external to the load, b) there is no need for an assumption concerning the internal connections of the load, its constituting receptors, or whether it is a passive or active load, thereby conferring great generality to the results, and c) operations tend to be easier than those involving impedances[3].

Some procedures have been proposed to balance three-wire three-phase loads, and to compensate the reactive power they absorb, using static VAr compensators. Several algorithms have been created to obtain the susceptances of the static VAr compensator. Those algorithms derive from methods deduced for passive loads consisting only of certain impedance connections[4-6]. They are deduced in this paper from power relations, thus showing that the solution is unequivocally valid not only for passive but also for active loads, whichever their internal connection might be.

\section{Relation between active and reactive powers delivered by each phase to a three-wire three-phase load}

The relation between the active and reactive powers delivered by each phase to a threewire three-phase load is the basis of the method shown here. Figure 1 represents a threephase load connected to a three-phase line with balanced voltages. If voltage $\bar{V}_{R}$ is chosen as reference phasor (fig. 1), then $\bar{V}_{R}=V \underline{10^{\circ}}, \bar{V}_{S}=V / \underline{1-120^{\circ}}, \bar{V}_{T}=V / 120^{\circ}$, and

$$
0=\bar{I}_{R}^{*}+\bar{I}_{S}^{*}+\bar{I}_{T}^{*}=\frac{P_{R}+\mathrm{j} Q_{R}}{V \underline{0^{\circ}}}+\frac{P_{S}+\mathrm{j} Q_{S}}{V \underline{I-120^{\circ}}}+\frac{P_{T}+\mathrm{j} Q_{T}}{V \underline{120^{\circ}}}
$$

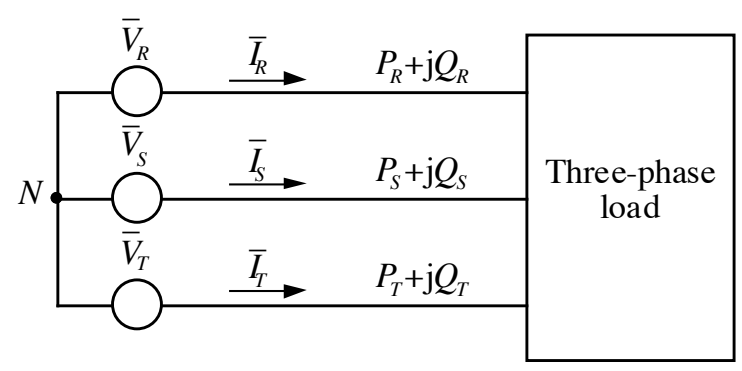

Fig. 1.- Power delivered to a three-wire three-phase load by each phase.

Where $\bar{I}_{R}^{*}$ is the conjugate of $\bar{I}_{R}$, and $P_{R}+\mathrm{j} Q_{R}=\bar{V}_{R} \bar{I}_{R}^{*}$ is the complex power delivered to the load through its $R$-phase. The same applies to the rest of the phases. As $1 / 120^{\circ}=-1 / 2+\mathrm{j} \sqrt{3} / 2$ and $1 \underline{-120^{\circ}}=-1 / 2-\mathrm{j} \sqrt{3} / 2$, from (1), two new equations of real numbers are obtained:

$$
\begin{aligned}
& 2 P_{R}-P_{S}-\sqrt{3} Q_{S}-P_{T}+\sqrt{3} Q_{T}=0 \\
& 2 Q_{R}-Q_{S}+\sqrt{3} P_{S}-Q_{T}-\sqrt{3} P_{T}=0
\end{aligned}
$$

These are the relations between the powers delivered by each phase looked for. Whichever the load connected to a three-wire three-phase line with balanced voltages might be, its powers must satisfy the relations given in (2). If

$$
Q_{R}=Q_{S}=Q_{T}
$$


one derives from (2) that

$P_{R}=P_{S}=P_{T}$

and the system is balanced. That is to say, it suffices to make the reactive powers delivered by the phases equal to each other to attain a balanced three-wire three-phase system. This is the basis of the method proposed in this paper, and used to obtain the algorithms of the compensator which balances any three-phase load: the method consists on a compensator, constituted entirely by reactances, which makes the reactive power delivered by the phases equal to each other. Thus, if $Q_{2}$ is the reactive power delivered to the set formed by the compensator and the load, then each phase delivers $Q=Q_{2} / 3$. The active power delivered now by each phase is $P$, and the active power delivered to the compensator-load group is $3 P$, which is also the active power delivered to the load, since no active power is delivered to the compensator as it consists of reactances only.

Thus, the new power factor is

$$
\cos \varphi_{2}=\frac{3 P}{\sqrt{(3 P)^{2}+(3 Q)^{2}}}=\frac{P}{\sqrt{P^{2}+Q^{2}}}
$$

If $Q_{2}=0$ is achieved, the power factor would be unity.

\section{Reactive power delivered by each phase to a three-wire purely-reactive load connected in triangle}

Let the powers $\mathrm{j} Q_{R S}, \mathrm{j} Q_{S T}$, and $\mathrm{j} Q_{T R}$, which are absorbed by the mono-phase reactive loads that constitute the triangle in figure 2 , be known. It is very useful for our purposes to find the relation between those powers and the complex powers delivered by each phase, which will be designated by $\bar{S}_{R}=P_{R}+\mathrm{j} Q_{R}, \bar{S}_{S}=P_{S}+\mathrm{j} Q_{S}$, and $\bar{S}_{T}=P_{T}+\mathrm{j} Q_{T}$.

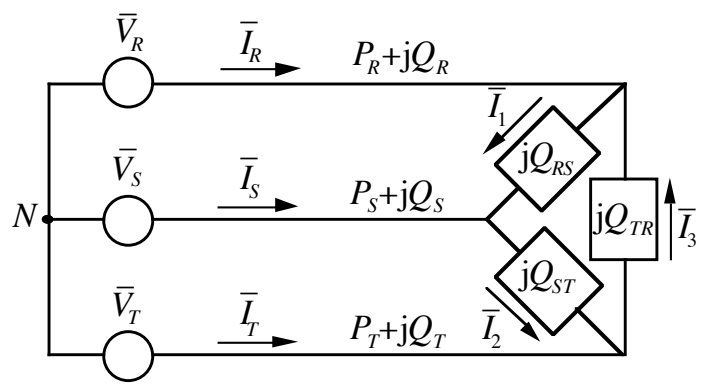

Fig. 2.- By choosing the appropriate value for $Q_{R S}, Q_{S T}$, and $Q_{T R}$, the reactive powers delivered by each phase, $Q_{R}, Q_{S}$, and $Q_{T}$, might be fixed at any desired value.

Seeing that $\bar{I}_{R}^{*}=\bar{I}_{1}^{*}-\bar{I}_{3}^{*}$, we have:

$\bar{I}_{R}^{*}=\frac{P_{R}+\mathrm{j} Q_{R}}{V / 0^{\circ}}=\frac{\mathrm{j} Q_{R S}}{\sqrt{3} V / 30^{\circ}}-\frac{\mathrm{j} Q_{T R}}{\sqrt{3} V / 150^{\circ}}=\frac{Q_{R S}-Q_{T R}}{2 \sqrt{3} V}+\mathrm{j} \frac{\sqrt{3}\left(Q_{R S}+Q_{T R}\right)}{2 \sqrt{3} V}$ 
Comparing the second and fourth members of this equation it is clear that

$$
Q_{R}=\frac{Q_{R S}+Q_{T R}}{2}
$$

Similarly

$$
\begin{aligned}
Q_{S} & =\frac{Q_{S T}+Q_{R S}}{2} \\
Q_{T} & =\frac{Q_{T R}+Q_{S T}}{2}
\end{aligned}
$$

The reactive powers delivered by each phase can be fixed at any desired value using three appropriate reactances, as shown in these last three equations. For instance, it is possible to achieve equal values for the three reactive powers delivered by the phases to a load, thus balancing it. Furthermore, all the reactive energy is compensated if those three powers are set to zero. This result will be used in a moment.

\section{Reactance compensator}

Figure 3 shows an unbalanced three-wire three-phase load. $Q_{R 1}, Q_{S 1}$ and $Q_{T 1}$ are the reactive powers delivered by each phase to the load; thus, the reactive power absorbed by the load is $Q_{1}=Q_{R 1}+Q_{S 1}+Q_{T 1}$ [7]. A reactance compensator is connected in a triangle configuration to balance phase currents. In order to do it, it is enough to make the reactive powers delivered by each phase to the compensator-load group equal, as seen before. That is to say, if $Q_{2}$ is the reactive power delivered to the compensator-load group, each phase must deliver $Q_{2} / 3$ to it. $Q_{2}$ can be any arbitrary value.

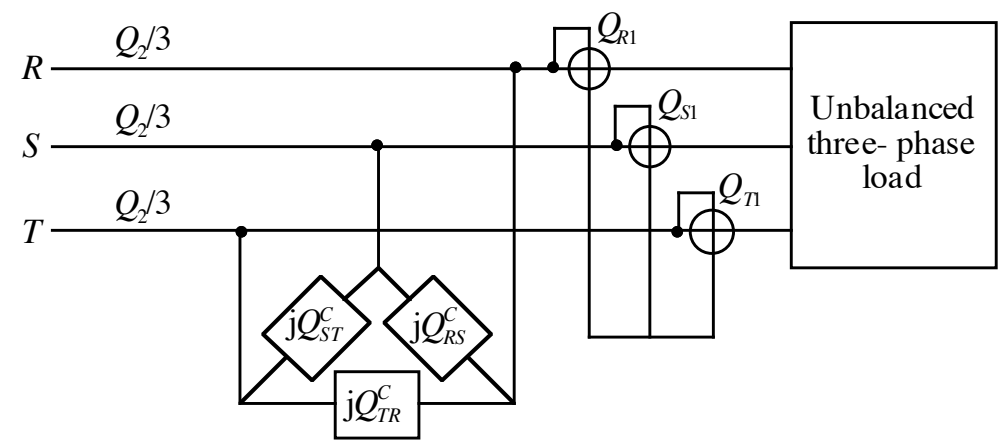

Fig. 3.- This compensator, formed by reactances, balances the system. Three varmeters measure $Q_{R 1}, Q_{S 1}$ and $Q_{T 1}$, and allow to obtain which power values the compensator's reactances must absorb.

The reactive powers of the compensator, $Q_{R S}^{C}, Q_{S T}^{C}$, and $Q_{T R}^{C}$, which make the reactive power delivered by each phase to the compensator-load group equal to $Q_{2} / 3$, will be determined. The power delivered to the compensator-load group by each phase is the sum of the power delivered to the load by each phase and the power delivered to the compensator. Taking into account (7), (8) and (9), this power is: 


$$
\begin{aligned}
& \frac{Q_{2}}{3}=Q_{R 1}+\frac{Q_{R S}^{C}+Q_{T R}^{C}}{2} \\
& \frac{Q_{2}}{3}=Q_{S 1}+\frac{Q_{S T}^{C}+Q_{R S}^{C}}{2} \\
& \frac{Q_{2}}{3}=Q_{T 1}+\frac{Q_{T R}^{C}+Q_{S T}^{C}}{2}
\end{aligned}
$$

The values looked for are obtained from this set of equations:

$$
\begin{aligned}
& Q_{R S}^{C}=\frac{Q_{2}}{3}+Q_{T 1}-Q_{R 1}-Q_{S 1} \\
& Q_{S T}^{C}=\frac{Q_{2}}{3}+Q_{R 1}-Q_{S 1}-Q_{T 1} \\
& Q_{T R}^{C}=\frac{Q_{2}}{3}+Q_{S 1}-Q_{T 1}-Q_{R 1}
\end{aligned}
$$

These are the reactive power values of the compensator's reactances that balance the three-phase system. The reactive power absorbed now is $Q_{2}$. If the only purpose is to balance the system without changing the value of the reactive power delivered to the compensator-load group, then we use $Q_{2}=Q_{1}=Q_{R 1}+Q_{S 1}+Q_{T 1}$. But if this reactive power, $Q_{2}$, is intended to be zero, then the powers of the reactances should be

$$
\begin{aligned}
& Q_{R S}^{C}=Q_{T 1}-Q_{R 1}-Q_{S 1} \\
& Q_{S T}^{C}=Q_{R 1}-Q_{S 1}-Q_{T 1} \\
& Q_{T R}^{C}=Q_{S 1}-Q_{T 1}-Q_{R 1}
\end{aligned}
$$

This are the values which balance the system and make the power factor unity. Under this conditions the power lost in the line is minimum.

The previous formulas constitute a very easy algorithm to deduce the values of the reactances of the compensator. The reactive powers delivered to the initial load by each phase can be measured using three VAr meters connected as shown in figure 3[7].

Knowing that $\sqrt{3} V$ is the effective value of the voltage between the phases of the load, the reactance values of the compensator are

$$
X_{R S}^{C}=\frac{3 V^{2}}{Q_{R S}^{C}}, X_{S T}^{C}=\frac{3 V^{2}}{Q_{S T}^{C}}, X_{T R}^{C}=\frac{3 V^{2}}{Q_{T R}^{C}}
$$

And susceptances are

$$
B_{R S}^{C}=-\frac{Q_{R S}^{C}}{3 V^{2}}, B_{S T}^{C}=-\frac{Q_{S T}^{C}}{3 V^{2}}, B_{T R}^{C}=-\frac{Q_{T R}^{C}}{3 V^{2}}
$$

The sign of each reactance is the same as the sign of its corresponding reactive power. For that reason, if the reactive power is positive, the corresponding reactance is 
inductive. If the power is negative, the corresponding reactance is capacitive. The sign of each susceptance is the opposite to that of its corresponding reactive power and reactance.

\section{Other algorithms}

Other algorithms can be deduced to obtain the reactive power values of the compensator's reactances. For instance, reactive powers of each phase can be obtained from the active and reactive powers measured as shown in figure 4[7]. In this figure, $\bar{S}_{R S 1}=P_{R S 1}+\mathrm{j} Q_{R S 1}$ and $\bar{S}_{T S 1}=P_{T S 1}+\mathrm{j} Q_{T S 1}$ are the powers acquired by the meters represented. As $\quad \bar{V}_{R}=V \underline{10^{\circ}}, \quad \bar{V}_{S}=V / \underline{1-120^{\circ}}=V(-1 / 2-\mathrm{j} \sqrt{3} / 2)$, $\bar{V}_{T}=V / 120^{\circ}=V(-1 / 2+\mathrm{j} \sqrt{3} / 2), \bar{I}_{R}^{L *}=I_{R}^{L} \underline{\varphi_{R}}$, and $\bar{I}_{T}^{L *}=I_{T}^{L} \underline{L^{\prime}-120^{\circ}+\varphi_{T}}$, then

$$
\begin{aligned}
& P_{R S 1}+\mathrm{j} Q_{R S 1}=\bar{U}_{R S} \bar{I}_{R}^{L *}=\left(\bar{V}_{R}-\bar{V}_{S}\right) \bar{I}_{R}^{L *}=\left(V / \underline{0^{\circ}}-V \underline{1-120^{\circ}}\right) I_{R}^{L} \underline{\varphi_{R}}= \\
& =\left(\frac{3}{2} P_{R 1}-\frac{\sqrt{3}}{2} Q_{R 1}\right)+\mathrm{j}\left(\frac{\sqrt{3}}{2} P_{R 1}+\frac{3}{2} Q_{R 1}\right)
\end{aligned}
$$

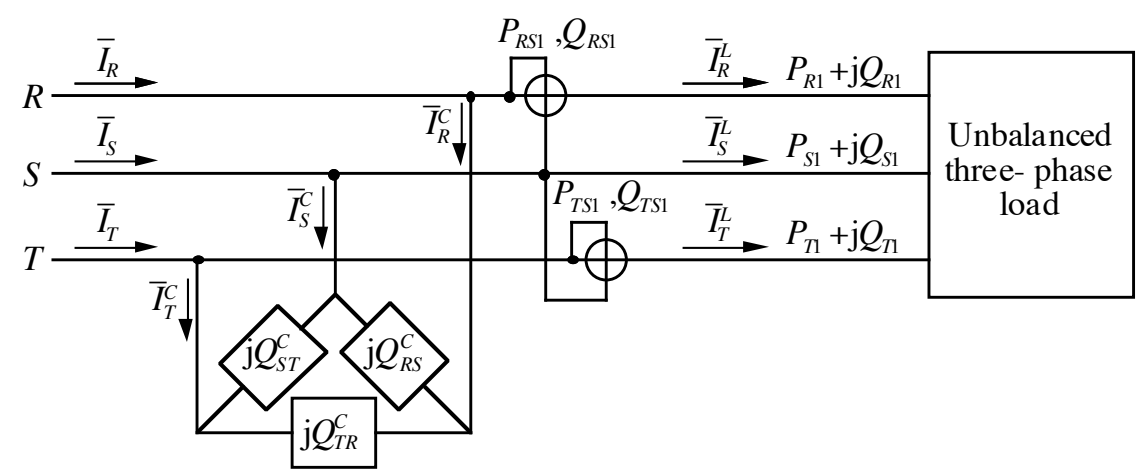

Fig. 4.- In order to use the measures from two wattmeters and two varmeters, other algorithms could be obtained.

If the first and last members are compared

$$
\begin{aligned}
P_{R S 1} & =\frac{3}{2} P_{R 1}-\frac{\sqrt{3}}{2} Q_{R 1} \\
Q_{R S 1} & =\frac{\sqrt{3}}{2} P_{R 1}+\frac{3}{2} Q_{R 1}
\end{aligned}
$$

By subtracting the second equation, previously multiplied by $\sqrt{3}$, from the first equation, $P_{R 1}$ is eliminated and the result is

$Q_{R 1}=-\frac{1}{2 \sqrt{3}} P_{R S 1}+\frac{1}{2} Q_{R S 1}$

Similarly 
$Q_{T 1}=\frac{1}{2 \sqrt{3}} P_{T S 1}+\frac{1}{2} Q_{T S 1}$

And, seeing that $Q_{R 1}+Q_{S 1}+Q_{T 1}=Q_{R S 1}+Q_{T S 1}$ [7], the value for $Q_{S 1}$ is

$$
Q_{S 1}=\frac{1}{2 \sqrt{3}}\left(P_{R S 1}-P_{T S 1}\right)+\frac{1}{2}\left(Q_{R S 1}+Q_{T S 1}\right)
$$

By substituting (18), (19), and (20) in (12), these values are obtained

$$
\begin{aligned}
& Q_{R S}^{C}=\frac{P_{T S 1}}{\sqrt{3}}-Q_{R S 1} \\
& Q_{S T}^{C}=-\frac{P_{R S 1}}{\sqrt{3}}-Q_{T S 1} \\
& Q_{T R}^{C}=\frac{P_{R S 1}-P_{T S 1}}{\sqrt{3}}
\end{aligned}
$$

These are the powers of the reactances of the compensator which are able to balance the currents and fully compensate the reactive power. If (18), (19), and (20) are substituted in (11), the compensator reactances, which balance the system, are obtained; nevertheless, the reactive energy absorbed in this case by the compensator-load group is $Q_{2}$, which can be arbitrarily fixed.

If $Q_{2}$ is the reactive power delivered to the load, the static compensator does not absorb reactive power (nor active power, of course).

\section{Example}

The meters in figure 4 show the following powers in $\mathrm{kW}$ and $\mathrm{kVAr}$ : $\bar{S}_{R S 1}=P_{R S 1}+\mathrm{j} Q_{R S 1}=4+\mathrm{j} 3$ and $\bar{S}_{T S 1}=P_{T S 1}+\mathrm{j} Q_{T S 1}=8+\mathrm{j} 5 . V=U / \sqrt{3}=220 \mathrm{~V} . U$ is the line-to-line voltage. Thus, load currents are

$$
\begin{aligned}
& \bar{I}_{R}^{L}=\left(\frac{\bar{S}_{R S 1}}{\bar{U}_{R S}}\right)^{*}=13.12 \underline{1-6.87^{\circ}} \\
& \bar{I}_{T}^{L}=\left(\frac{\bar{S}_{T S 1}}{\bar{U}_{T S}}\right)^{*}=24.76 \underline{157.99^{\circ}} \\
& \bar{I}_{S}^{L}=-\bar{I}_{R}^{L}-\bar{I}_{T}^{L}=32.57 \underline{1-143.39^{\circ}}
\end{aligned}
$$

The reactive power delivered to the load is $Q_{1}=Q_{R S 1}+Q_{T S 1}=8 \mathrm{kVAr}$

If the objective is to balance the system without altering the reactive power it delivers to the load, (21) should be used by adding $Q_{2} / 3=Q_{1} / 3=8 \times 10^{3} / 3$ to them:

$Q_{R S}^{C}=\frac{Q_{2}}{3}+\frac{P_{T S 1}}{\sqrt{3}}-Q_{R S 1}=4285.47 \mathrm{VAr}$ 


$$
\begin{aligned}
& Q_{S T}^{C}=\frac{Q_{2}}{3}-\frac{P_{R S 1}}{\sqrt{3}}-Q_{T S 1}=-4642.73 \mathrm{VAr} \\
& Q_{T R}^{C}=\frac{Q_{2}}{3}+\frac{P_{R S 1}-P_{T S 1}}{\sqrt{3}}=357.27 \mathrm{VAr}
\end{aligned}
$$

(14) gives the values of the susceptances:

$$
\begin{aligned}
& B_{R S}^{C}=-\frac{Q_{R S}^{C}}{3 V^{2}}=-29.51 \times 10^{-3} \mathrm{~S} \\
& B_{S T}^{C}=-\frac{Q_{S T}^{C}}{3 V^{2}}=31.97 \times 10^{-3} \mathrm{~S} \\
& B_{T R}^{C}=-\frac{Q_{T R}^{C}}{3 V^{2}}=-2.46 \times 10^{-3} \mathrm{~S}
\end{aligned}
$$

The phase currents of the compensator are

$$
\left[I^{C}\right]=\left[\begin{array}{l}
\bar{I}_{R}^{C} \\
\bar{I}_{S}^{C} \\
\bar{I}_{T}^{C}
\end{array}\right]=\left[\begin{array}{ccc}
\mathrm{j}\left(B_{R S}^{C}+B_{T R}^{C}\right) & -\mathrm{j} B_{R S}^{C} & -\mathrm{j} B_{T R}^{C} \\
-\mathrm{j} B_{R S}^{C} & \mathrm{j}\left(B_{R S}^{C}+B_{S T}^{C}\right) & -\mathrm{j} B_{S T}^{C} \\
-\mathrm{j} B_{T R}^{C} & -\mathrm{j} B_{S T}^{C} & \mathrm{j}\left(B_{T R}^{C}+B_{S T}^{C}\right)
\end{array}\right]\left[\begin{array}{c}
\bar{V}_{R} \\
\bar{V}_{S} \\
\bar{V}_{T}
\end{array}\right]=\left[\begin{array}{c}
11.74 \underline{1-63.96^{\circ}} \\
11.74 \underline{156.04^{\circ}} \\
11.74 \underline{176.04^{\circ}}
\end{array}\right]
$$

Which is a negative-sequence symmetrical current set (more comments on this result will come later). The phase currents before the compensator are

$$
[I]=\left[\begin{array}{l}
\bar{I}_{R} \\
\bar{I}_{S} \\
\bar{I}_{T}
\end{array}\right]=\left[\begin{array}{c}
\bar{I}_{R}^{L} \\
\bar{I}_{T}^{L} \\
\bar{I}_{S}^{L}
\end{array}\right]+\left[\begin{array}{l}
\bar{I}_{R}^{C} \\
\bar{I}_{S}^{C} \\
\bar{I}_{T}^{C}
\end{array}\right]=\left[\begin{array}{c}
21.85 \underline{1-33.69^{\circ}} \\
21.85 \underline{1-153.69^{\circ}} \\
21.85 \underline{86.31^{\circ}}
\end{array}\right]
$$

Which is a positive-sequence symmetrical current set, as intended.

If $[V]^{t}=\left[\bar{V}_{R}, \bar{V}_{S}, \bar{V}_{T}\right]$, then the complex power delivered to the compensator-load group is[7]

$$
[V]^{t}[I]^{*}=12000+\mathrm{j} 8000
$$

which is equal to that of the load alone.

If the objective is to compensate the reactive power, then $Q_{2}=0$. In that case

$$
\begin{aligned}
& B_{R S}^{C}=-11.15 \times 10^{-3} \mathrm{~S}, B_{S T}^{C}=50.34 \times 10^{-3} \mathrm{~S}, \text { and } B_{T R}^{C}=15.91 \times 10^{-3} \mathrm{~S} ; \\
& {[I]=\left[\begin{array}{l}
\bar{I}_{R} \\
\bar{I}_{S} \\
\bar{I}_{T}
\end{array}\right]=\left[\begin{array}{c}
18.18 \underline{0^{\circ}} \\
18.18 \underline{\frac{1-120^{\circ}}{18.18} \underline{120^{\circ}}}
\end{array}\right]}
\end{aligned}
$$


and the complex power delivered to the compensator-load group is

$[V]^{t}[I]^{*}=12000+\mathrm{j} 0$

\section{Currents of the compensator}

The previous example showed that the currents of the compensator form a negativesequence symmetrical current set, when only the line currents are balanced without compensating the reactive power. This is not accidental, for it happens whenever the value of the compensator's reactive energy is zero. Indeed, as the phase-neutral voltages are balanced, they only have positive-sequence symmetrical component $\bar{V}_{d}$, therefore only the currents' positive-sequence symmetrical component of the compensator $\bar{I}_{d}^{C}$ intervenes in the formula of the power it absorbs. That is to say, the value of the complex power supplied to the compensator at all times is

$$
S^{C}=3 \bar{V}_{d} \bar{I}_{d}^{C *}=P^{C}+\mathrm{j} Q^{C}
$$

where $\bar{I}_{d}^{C *}$ is the conjugate of $\bar{I}_{d}^{C}$. The value of the active power $P^{C}$ is always zero, due to the compensator being formed only by reactances. If the value of the reactive power $Q^{C}$ is also zero, then

$$
S^{C}=3 \bar{V}_{d} \bar{I}_{d}^{C *}=0
$$

Seeing that $\bar{V}_{d} \neq 0$, it follows that $\bar{I}_{d}^{C *}=0$ and $\bar{I}_{d}^{C}=0$.

Therefore, if $Q^{C}=0$, the compensator's currents lack of positive-sequence symmetrical component. Those currents are also lacking zero-sequence symmetrical component, seeing that they add up to zero, consequently only the negative-sequence symmetrical component cannot be zero. It is concluded that the compensator's currents are a negative-sequence symmetrical current set when the value of its reactive power is zero.

\section{Compensator for four-wire three-phase loads}

Figure 5 shows a procedure to cancel out the current through the neutral wire of any four-wire three-phase load. Two reactances, $X_{1}$ and $X_{2}$, are connected between the $R$ and $S$ phases, and the neutral wire, respectively, so that

$$
-\frac{V / 0^{\circ}}{\mathrm{j} X_{1}}-\frac{V /-120^{\circ}}{\mathrm{j} X_{2}}=\bar{I}_{N}
$$




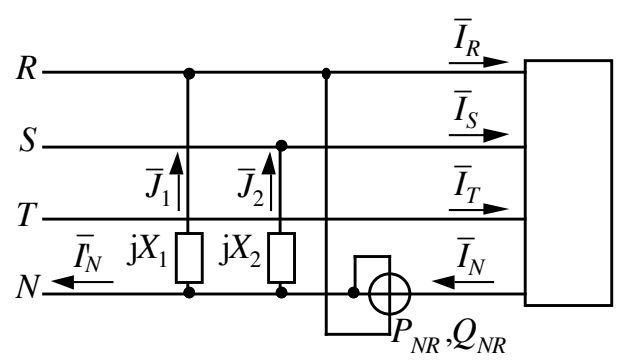

Fig. 5.- If $\bar{J}_{1}+\bar{J}_{2}=\bar{I}_{N}$, then $\bar{I}_{N}^{\prime}$ is zero, and the four-wire load behaves as a three-wire load.

Voltages of the initial load are not affected by the connection of $X_{1}$ and $X_{2}$. The active power is not increased either. Nevertheless, the current through the neutral wire, $\bar{I}_{N}^{\prime}$, is canceled out, which, in fact, transforms the load into a three-wire three-phase load. The previously seen compensator can now be connected to the three phases, as shown in Figure 6.

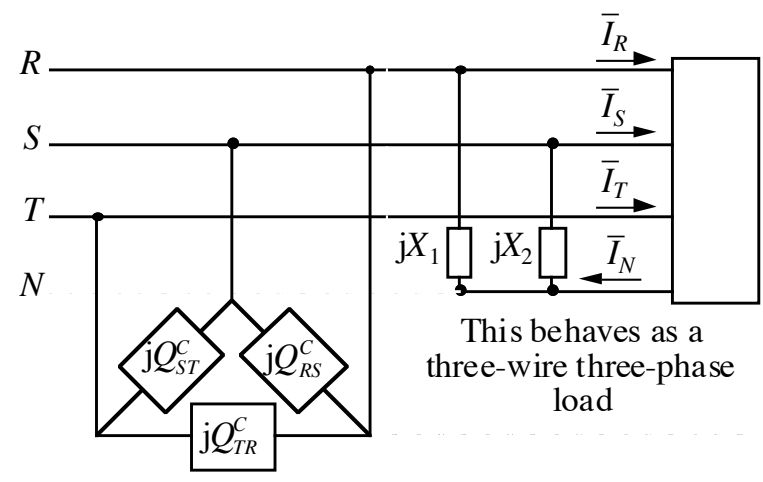

Fig. 6.- Static VAr compensator for a four-wire three-phase load.

Phasor $\bar{I}_{N}$ can be determined by using a watt-varmeter connected as indicated in figure 5. This meter reads

$$
\left(-\bar{V}_{R}\right)\left(-\bar{I}_{N}\right)^{*}=\bar{V}_{R} \bar{I}_{N}^{*}=P_{N R}+\mathrm{j} Q_{N R}
$$

where $P_{N R}$ and $Q_{N R}$ are the wattmeter and varmeter readings, respectively. From this formula it can be seen that

$$
\bar{I}_{N}=\left(\frac{P_{N R}+\mathrm{j} Q_{N R}}{\bar{V}_{R}}\right)^{*}=\frac{P_{N R}}{V}-\mathrm{j} \frac{Q_{N R}}{V}
$$

If this result is substituted in (22), then

$$
\begin{gathered}
X_{1}=\frac{\sqrt{3} V^{2}}{P_{N R}-\sqrt{3} Q_{N R}} \\
X_{2}=\frac{\sqrt{3} V^{2}}{2 P_{N R}}
\end{gathered}
$$


which are the reactances that cancel out the current through the neutral wire.

\section{Load voltage}

So far, the voltages of the load have been supposed to be symetrical. But, if the load currents are not balanced, then the voltage drops of the phases, $\bar{Z}^{L} \bar{I}_{R}, \bar{Z}^{L} \bar{I}_{S}$, and $\bar{Z}^{L} \bar{I}_{T}$, are different, which leads to unbalanced voltages near the load. However, the iterative operation of the compensator quickly produces symmetrical voltages, when connected.

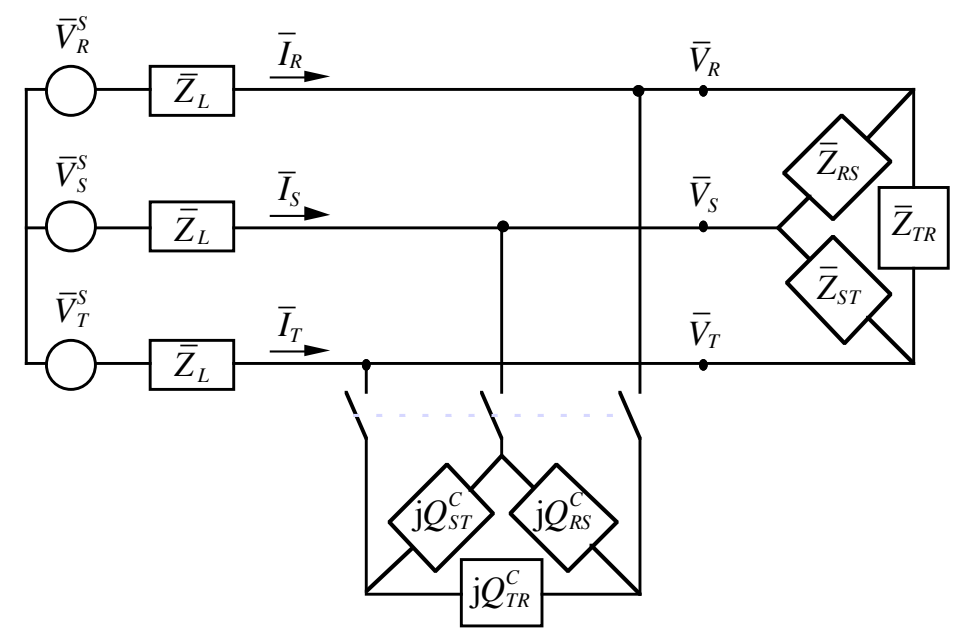

Fig. 7.- Simulation network.

Figure 7 summarizes one of the simulations made. It shows a three-wire three-phase system formed by three symmetrical voltage sources $\bar{V}_{R}^{S}=100 \underline{0^{\circ}}, \bar{V}_{S}^{S}=100 \underline{1-120^{\circ}}$, and $\bar{V}_{T}^{S}=100 / 120^{\circ}$. The impedance of each phase wire is $\bar{Z}^{L}=1+\mathrm{j} 1$. The load is a triangle of impedances $\bar{Z}_{R S}=10+\mathrm{j} 15, \bar{Z}_{S T}=20+\mathrm{j} 25$, and $\bar{Z}_{T R}=80+\mathrm{j} 30$. The effective values of the phase currents without the compensator are

$I_{R}=9.74 \mathrm{~A}, I_{S}=10.84 \mathrm{~A}$, and $I_{T}=5.53 \mathrm{~A}$.

The load voltages are

$V_{R}=86.70 \mathrm{~V}, V_{S}=85.42 \mathrm{~V}$, and $V_{T}=92.67 \mathrm{~V}$.

Table 1 shows the values of the phase currents and voltages when the compensator is connected, which have been obtained from the iterated use of (12) and (13). $V$ has been substituted each time by $U_{R S} / \sqrt{3}$ in these algorithms. After the fifth iteration of the compensator the currents and voltages are balanced. The tendency to balance is fast, because right after the second iteration of the algorithm the effective value of the currents are very close to each other, as occurs with the effective values of the voltages.

\section{Experimental test}

An experimental automatic device was designed and built at our laboratory to test the results of this paper. Figure 8 shows its block diagram. It consists of a passive load 
formed by impedances connected between every two phases, which is represented in figure 8 by the unbalanced three-phase load block. The connection of impedances can be modified manually, thus allowing to obtain balanced or unbalanced loads easily. Using a Data Acquisition (DAQ) card and the application LabVIEW running on a desktop computer, the instantaneous currents $i_{R}$ and $i_{T}$, and the instantaneous voltages $v_{R S}$ and $v_{T S}$, are measured before and after the compensator. So, by programming the appropriate algorithms in LabVIEW, the values of the reactances of the compensator are obtained and the instruction to connected them to the original load is given through a digital output card.

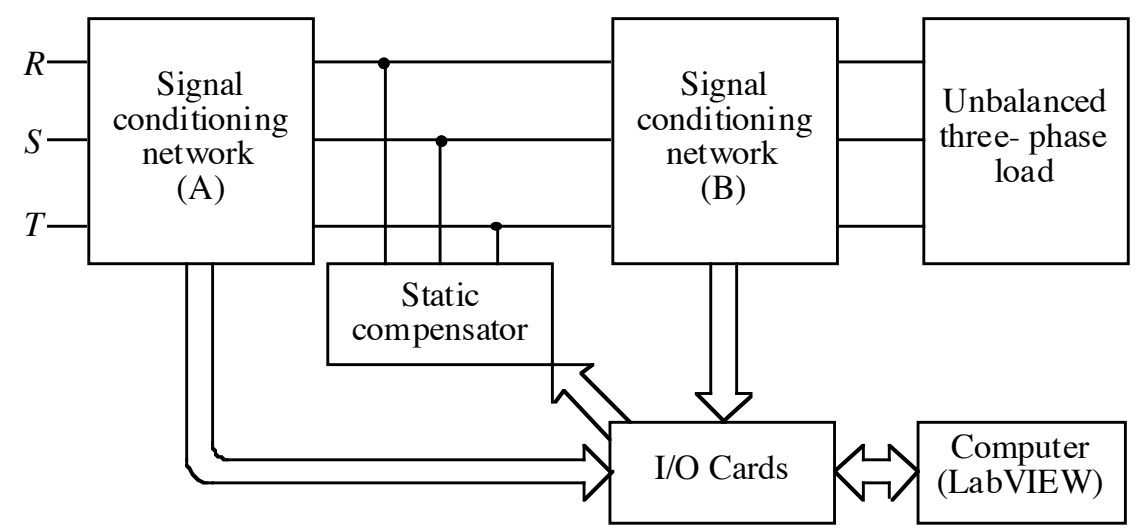

Fig. 8.- Block diagram of the experimental device.

Figure 9 is a screenshot from the tests. The upper part of the picture shows the unbalanced currents near the load, and the lower part shows the balanced currents resulting from using the compensator.

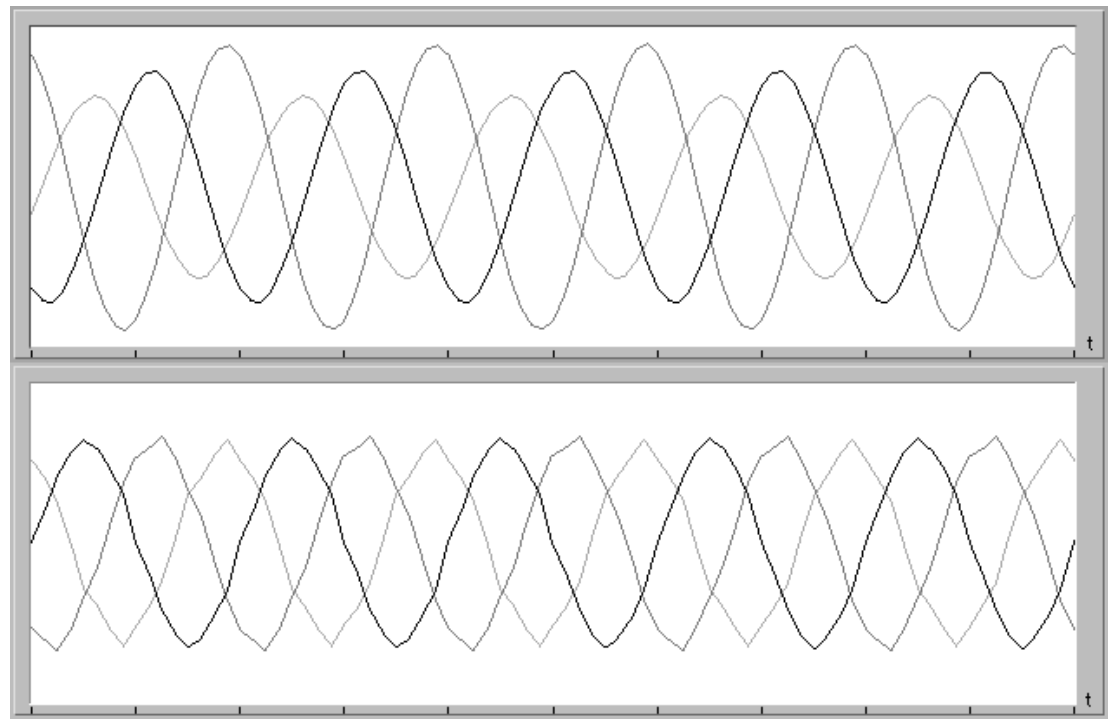

Fig. 9.- Screenshots showing the unbalanced phase currents near the load (top) and the balanced currents resulting from using the compensator (bottom). 


\section{Conclusion}

Advantages of the analysis of three-phase loads described by relations between powers, instead of impedances, are shown in this paper. When its use is possible, this analysis method explicitly indicates if results can, or cannot, be applied to active loads. More specifically, the possibility to use it to obtain the static VAr compensators' algorithms is shown. Then, it is made clear that the compensators' function is valid whatever kind of three-phase load it is connected to, particularly, whether they are active or passive loads. It is also shown that, if a compensator does not deliver reactive power, its three currents are always a negative-sequence symmetrical current set.

\section{References}

[1] Nilsson, James W, Riedel, Susan, Electric Circuits, $7^{\text {th }}$ Edition, Prentice Hall, 2005.

[2] A. M. Howatson, Electric Circuits and Systems, Oxford University Press, Oxford, 1996.

[3] F. R. Quintela, R. C. R. Melchor and M. M. R. Melchor, Power of balanced polyphase systems: an application of the multi-terminal network power theorem, Int. J. Elect. Enging. Educ. 42/4 October 2005, pp. 325-337.

[4] San-Yi Lee, Wei-Nan Chang, Chi-Jui Wu, A compact algorithm for three-phase three-wire system reactive power compensation and load balancing, International Conference on Energy Management and Power Delivery, 1995. Proceedings of EMPD '95., 1995. Volume 1 Nov. 1995, pag. 358-363

[5] Bhim Singh, Anuradha Saxena, D. P. Kothari, Power factor correction and load balancing in three-phase distribution systems, TENCON '98. 1998 IEEE Region 10 International Conference on Global Connectivity in Energy, Computer, Communication and Control. 2 (1998) 479-488.

[6] S. Lee, C. J. Wu, W. N. Chang, A compact control algorithm for reactive power compensation and load balancing with static Var compensator, Electr. Power Syst. Res. 58 (2001) 63-70.

[7] F. Redondo Quintela and N. Redondo Melchor, Multi-terminal network power measurement, Int. J. Elect. Enging. Educ. April 2002, pp. 148-161. 